\title{
IMPROVED LINEARIZATION OF THE OPTIMAL COMPRESSION FUNCTION FOR LAPLACIAN SOURCE
}

\author{
Zoran H. Perić - Lazar Z-. Velimirović - Milan R. Dinčić *
}

\begin{abstract}
In this paper, linearization of the optimal compression function is done and hierarchical coding (by coding the regions firstly and then the cells inside the region) is applied, achieving simple and fast process of coding and decoding. The signal at the entrance of the scalar quantizer is modeled by Laplacian probability density function. It is shown that the linearization of inner regions very little influences distortion and therefore only the last region should be optimized. Two methods of optimization of the last region are proposed, that improve performances of the scalar quantizer, and obtained SQNR (signal-to-quantization noise ratio) is close to that of the nonlinear optimal compression function.
\end{abstract}

K e y w or d s: linearization, optimal compression function, scalar compandor

\section{INTRODUCTION}

With the scalar quantization process, the current value of continuous input signal rounds up to the nearest allowed value from the finite set of discrete amplitude levels. Scalar quantizer is unambiguously determined with the set of allowable output amplitude levels, called representational levels, and with division of the values of input range on cells or quantization intervals. Quantizer can be uniform (all the quantization intervals are of the same width) and nonuniform (different width of the quantization intervals) [1]. Uniform quantizers are suitable for signals that have approximately uniform distribution. How most of the signals do not have a uniform distribution (usually small current values are more likely than the large ones), there is a need for using nonuniform quantizer. One of the most used methods for the realization of the nonuniform quantizer is companding technique, in which a specific compressor function is applied on an input signal. The most often used compressor functions are optimal compressor function (which gives the maximum signal-to-quantization noise ratio $S Q N R$ for the reference variance of the input signal) and a logarithmic $A$-law and $\mu$-law compression functions, by which maximum $S Q N R$ cannot be achieved, but that provides a constant $S Q N R$ in wide range of an input signal variance [1]. These compressor functions are very complicated to be realized practically. Therefore, in order to achieve easier practical realization, linearization of the optimal compressor function is performed. Thus linearization of $A$-law and $\mu$-law, done by defining a well known segment $A$-law and segment $\mu$-law compression functions [1], where input range of quantizer is divided into segment $s$ and inside each segment a linear compressor function is used, ie uniform segment division on cells is done. The number of cells in each segment is equal and hierarchical coding can be applied, which means that firstly the segment is coded and then the cells inside the segment. This way the piecewise uniform quantizer is obtained. The piecewise uniform scalar quantizer is analyzed in [2]. By the algorithm realization for the speech signal [2], not only that the higher quality signal than a quality defined by standard G.711 is obtained, but the bit-rate reduces for about $1 \mathrm{bit} /$ samples. The linearization of the optimal compressor function is done in $[3,4]$. In [3], the linearization with unequal number of cells per region is done, ie for each segment, the optimization of number of cells is done. The disadvantage of this method is a high complexity of quantizer, the complexity of coding and decoding, and the impossibility to apply hierarchical coding. The analysis of compressor function for Laplacian source is shown in [4].

In this paper, the linearization of the optimal compressor function for the input signal with Laplacian distribution is done, in the similar way as for segment $A$-law and segment $\mu$-law compression function. All segments have the same number of cells and a hierarchical coding is done. It is shown that the highest quality of the output signal is achieved in the case when the last representational level is determined from the centroid condition. The process of designing scalar quantizer whose representational levels are determined from centroids' conditions, for Laplacian and Gaussian source, is described in [5]. The linearization of the optimal compressor function proposed in this paper is much simpler than in [2-4] and the results, regarding the quality of the output signals, are better. The special contribution of this work represents the optimization of the last region, by which the improvement is achieved comparing to previous linearization techniques. Two methods of the last region optimization are presented and obtained values of $S Q N R$ are close to those values of $S Q N R$ for nonlinear optimal compressor function.

* Faculty of Electronic Engineering Nis, Aleksandra Medvedeva 14, 18000 Nis, Serbia; velimirovic.lazar@gmail.com 


\section{OPTIMAL NONLINEAR COMPRESSOR FUNCTION}

The scalar quantizer is determined by the representational levels $\left\{y_{1}, \ldots, y_{N}\right\}$ and decision thresholds $\left\{t_{0}, t_{1}, \ldots, t_{N}\right\}$. The input range of the quantizer is divided into $N$ cells or quantization intervals $\alpha_{j}=$ $\left[t_{j-1}, t_{j}\right), j=1,2, \ldots, N$. During the quantization, the quantization error, expressed with distorsion, is made. The total distortion can be found as a sum of the granular $D_{g}$ and the overload $D_{o}$ distortion [1]

$$
D=D_{g}+D_{o}
$$

that is determined as follows

$$
\begin{aligned}
& D_{g}=\sum_{j=2}^{N-1} \int_{t_{j-1}}^{t_{j}}\left(x-y_{j}\right)^{2} p(x) \mathrm{d} x, \\
& D_{o}=2 \int_{t_{N-1}}^{\infty}\left(x-y_{N}\right)^{2} p(x) \mathrm{d} x,
\end{aligned}
$$

where $p(x)$ is Laplacian PDF (probability density function) which is defined as

$$
p(x)=\frac{1}{\sqrt{2} \sigma} e^{-\sqrt{2}|x| / \sigma} .
$$

Since $p(x)$ is an even function, the quantizer will be symmetrical. The decision thresholds and representational levels in the negative section of the real axis will be symmetrical to those in the positive section of the real axis. Therefore, only the positive section of the real axis will be considered. One of the methods of the realization of the nonuniform quantization is companding technique. Nonuniform quantization can be achieved by compressing the signal $\mathrm{x}$ using a nonuniform compressor characteristic $c(\cdot)$, by quantizing the compressed signal $c(x)$ employing a uniform quantizer, and by expanding the quantized version of the compressed signal using a nonuniform transfer characteristic $c^{-1}(\cdot)$ that is inverse to that of the compressor. The granular distortion for the companding quantizer is presented in the form of Bennett's integral as $[1,7-8]$

$$
D_{g}=\frac{1}{12 N^{2}} \int_{R} \frac{p(x)}{[\lambda(x)]^{2}} \mathrm{~d} x,
$$

where $\lambda(x)$ is the density of representational level and is defined as

$$
\lambda(x)=\frac{1}{N \Delta_{i}}, \quad x \in\left(t_{i-1}, t_{i}\right]
$$

and $\Delta_{i}=t_{i}-t_{i-1}$ denotes amplitude quantum size. Bennett's integral (5) obtains the form

$$
D_{g}=\frac{1}{12} \int_{R} \Delta_{i}^{2} p(x) \mathrm{d} x .
$$

The minimum of the Bennett's integral (5) is also a minimum of the distortion of the nonuniform scalar quantizer, which means that by using the companding technique, optimal scalar quantization can be realized. By determination of the gradient of the compressor function (for positive characteristic's section)

$$
C^{\prime}(x)=\frac{\Delta}{\Delta_{i}}=\frac{2 x_{\max }}{N \Delta_{i}} \approx c^{\prime}\left(y_{i}\right), i=N / 2+1, \ldots, N,
$$

an expression for the density of representational level is achieved, and with substitution in (5), a known form of Bennett's integral is obtained

$$
D_{g}=\frac{x_{\max }^{2}}{3 N^{2}} \int_{R} \frac{p(x)}{\left[c^{\prime}(x)\right]^{2}} \mathrm{~d} x .
$$

The performances of the quantizer are determined by $S Q N R$ which is defined as follows [1]

$$
S Q N R=10 \log _{10} \frac{\sigma^{2}}{D}(\mathrm{~dB}) .
$$

The optimal compressor function $c(x)$ by which the maximum $S Q N R$ is achieved for the reference variance of an input signal is defined as [1]

$$
c(x)=x_{\max } \frac{\int_{0}^{x} p^{1 / 3}(x) \mathrm{d} x}{\int_{0}^{x_{\max }} p^{1 / 3}(x) \mathrm{d} x} .
$$

Without diminishing the generality, the quantizer design will be done for the reference input variance of $\sigma_{\text {ref }}^{2}=1$.

Based on the equations

$$
c\left(t_{i}\right)=-x_{\max }+i \frac{2 x_{\max }}{N}, c\left(y_{i}\right)=-x_{\max }+\left(i-\frac{1}{2}\right) \frac{2 x_{\max }}{N} .
$$

The next expressions for the decision thresholds $t_{i}$ and for representational levels $y_{i}, i=N / 2+1, \ldots, N$, are obtained

$$
\begin{aligned}
& t_{i}=\frac{3}{\sqrt{2}} \ln \frac{N}{2 N-2 i+(2 i-N) \exp \frac{-\sqrt{2} x_{\max }}{3}} \\
& y_{i}=\frac{3}{\sqrt{2}} \ln \frac{N}{2 N-2 i+1+(2 i-N-1) \exp \frac{-\sqrt{2} x_{\max }}{3}}
\end{aligned}
$$

In this paper, the design of the scalar quantizer is performed for the case when the maximum amplitude of the quantizer is finite. The dependency of the maximum amplitude of the quantizer on the number of representational levels is shown in [6]. In [3], it is shown that its optimum value in that case is equal to

$$
x_{\max }=\frac{3}{\sqrt{2}} \ln (N+1) .
$$


Table 1. Comparing granular distortion to ranges for different quantizer models

\begin{tabular}{lcccccccc}
\hline & $\left(-t_{9}^{*}, t_{9}^{*}\right)$ & $\left(-t_{10}^{*}, t_{10}^{*}\right)$ & $\left(-t_{11}^{*}, t_{11}^{*}\right)$ & $\left(-t_{12}^{*}, t_{12}^{*}\right)$ & $\left(-t_{13}^{*}, t_{13}^{*}\right)$ & $\left(-t_{14}^{*}, t_{14}^{*}\right)$ & $\left(-t_{15}^{*}, t_{15}^{*}\right)$ & $\left(-t_{16}^{*}, t_{16}^{*}\right)$ \\
\hline$D_{g}^{\text {Ben }}$ & $3.354 \times 10^{-5}$ & $6.708 \times 10^{-5}$ & $1.006 \times 10^{-4}$ & $1.342 \times 10^{-4}$ & $1.677 \times 10^{-4}$ & $2.012 \times 10^{-4}$ & $2.348 \times 10^{-4}$ & $2.683 \times 10^{-4}$ \\
$D_{g}^{\text {exact }}$ & $3.354 \times 10^{-5}$ & $6.708 \times 10^{-5}$ & $1.006 \times 10^{-4}$ & $1.342 \times 10^{-4}$ & $1.677 \times 10^{-4}$ & $2.012 \times 10^{-4}$ & $2.348 \times 10^{-4}$ & $2.682 \times 10^{-4}$ \\
$D_{g}^{P U}$ & $3.369 \times 10^{-5}$ & $6.742 \times 10^{-5}$ & $1.012 \times 10^{-4}$ & $1.351 \times 10^{-4}$ & $1.694 \times 10^{-4}$ & $2.043 \times 10^{-4}$ & $2.415 \times 10^{-4}$ & $3.491 \times 10^{-4}$ \\
\hline
\end{tabular}

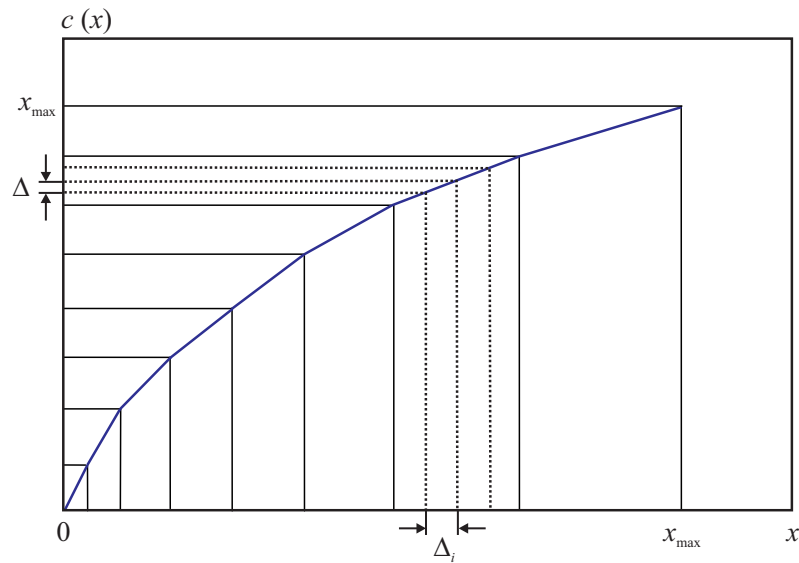

Fig. 1. Piecewise linear compressor functions illustration

\section{LINEARIZATION OF THE OPTIMAL COMPRESSION FUNCTION}

This section contains a detailed description of linearization of the optimal compression function. The linearization is done in the way that the amplitude quantizer range is divided into $L$ regions, and each region is uniformly divided into $N / L$ cells of equal width. The piecewise uniform scalar quantizer is obtained in this way. Due to the symmetry of quantizer, we will only consider the positive region of characteristic (see Figure 1). The decision thresholds between regions in the positive section of the characteristics $t_{i}^{*}, i=L / 2+1, \ldots, L$ are determined by using the expression

$$
t_{i}^{*}=\frac{3}{\sqrt{2}} \ln \frac{L}{2 L-2 i+(2 i-L) \exp \left(-\frac{\sqrt{2} x_{\max }^{*}}{3}\right)},
$$

where $x_{\max }^{*}$ denotes the maximum amplitude of the linearized piecewise uniform scalar quantizer. The size of the cells in the $i$-th region is equal to

$$
\Delta_{i}^{*}=\frac{t_{i}^{*}-t_{i-1}^{*}}{N / L}
$$

With $t_{i, j}, i=1, \ldots, L, j=0, \ldots, N / L$ the threshold point between cells in the $i$-th region is denoted, where $t_{i, 0}=t_{i-1}^{*}$ and $t_{i, N / L}=t_{i}^{*}$. With $y_{i, j}, i=1, \ldots, L$, $j=1, \ldots, N / L$ representational level the $j$-th cell into the $i$-th region is denoted. Due to the uniform regions of the cell division it goes

$$
t_{i, j}=t_{i-1}^{*}+j \Delta_{i}^{*}, \quad y_{i, j}=t_{i-1}^{*}+(j-1 / 2) \Delta_{i}^{*} .
$$

Now, we want to investigate how much the granular distortion of the piecewise uniform (PU) quantizer is higher than the granular distortion of the optimal companding (OC) quantizer, and how much each region contributes on this increase of the granular distortion. Therefore, we will calculate the granular distortion both of the PU quantizer and of the OC quantizer and compare them. To find the contribution of each region on the increase of the granular distortion, the granular distortions of PU and OC quantizers will be calculated for intervals $\left[-t_{i}^{*}, t_{i}^{*}\right] \equiv\left[t_{L / 2-i}^{*}, t_{i}^{*}\right], i=L / 2+1, \ldots, L$. The granular distortion of the PU quantizer in the interval $\left[-t_{i}^{*}, t_{i}^{*}\right]$, denoted as $D_{g}^{P U}\left(t_{i}^{*}\right)$, is defined as

$$
D_{g}^{P U}\left(t_{i}^{*}\right)=2 \sum_{k=1}^{i} \frac{\Delta_{k}^{* 2}}{12} P_{k}, \quad i=L / 2+1, \ldots, L
$$

where $P_{k}$ is the probability that the current value of amplitude input signal belongs to the $k$-th region $\left[t_{k-1}^{*}, t_{k}^{*}\right]$

$$
P_{k}=\int_{t_{k-1}^{*}}^{t_{k}^{*}} p(x) \mathrm{d} x=\frac{1}{2}\left(\exp \left(-t_{k-1}^{*}\right)-\exp \left(-t_{k}^{*}\right)\right)
$$

The granular distortion of the OC quantizer in the interval $\left[-t_{i}^{*}, t_{i}^{*}\right]$ can be calculated in two ways. The first way is by analytical solving of Bennett's integral given with the equation (5), where the optimal compressor function $\mathrm{c}(\mathrm{x})$ is determined with (11) and the maximum amplitude of the quantizer is given by (15). We obtain the following expression for the granular distortion in the interval $\left[-t_{i}^{*}, t_{i}^{*}\right]$

$$
D_{g}^{B e n}\left(t_{i}^{*}\right)=\frac{9}{2 N^{2}}\left(1-\exp \frac{-\sqrt{2} t_{i}^{*}}{3}\right), \quad i=\frac{L}{2}+1, \ldots, L .
$$

The second way to calculate the granular distortion of the OC quantizer is to use the following exact expression [1]

$$
\begin{gathered}
D_{g}^{\text {exact }}\left(t_{i}^{*}\right)=2 \sum_{k=L / 2+1}^{i} \sum_{j=1}^{N / L} \int_{t_{k, j-1}}^{t_{k, j}}\left(x-y_{k, j}\right)^{2} p(x) \mathrm{d} x \\
i=L / 2+1, \ldots, L
\end{gathered}
$$

In Table 1, numerical values of the previous distortions are given for the quantizer with $N=128$ levels and $L=16$ regions. We can see that in the intervals $\left[-t_{i}^{*}, t_{i}^{*}\right]$, $i=9, \ldots, 15$, the granular distortion of the PU quantizer, 


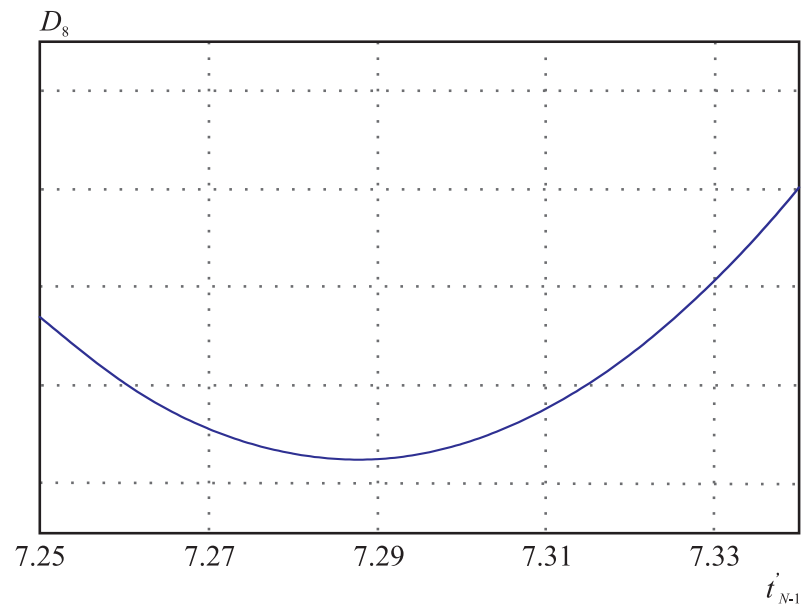

Fig. 2. Numerical determination of the decision threshold of the granular region

Table 2. Key parameters and performances of the proposed quantizer model

\begin{tabular}{ccccc}
\hline method & $t_{\max }$ & $t_{N-1}^{\prime}$ & $\Delta_{8}$ & $S Q N R$ \\
\hline$I$ & 10.31 & 9.56 & 0.751 & 34.56 \\
$I I$ & 10.31 & 7.29 & 0.427 & 35.34 \\
\hline
\end{tabular}

Table 3. Quantizer performances depending on number of levels and number of regions for method II

\begin{tabular}{ccccc}
\hline & $N=16$ & $N=32$ & $N=64$ & $N=128$ \\
\hline$L=8$ & 17.91 & 23.49 & 29.10 & 34.68 \\
$L=16$ & - & 23.74 & 29.54 & 35.34 \\
$L=32$ & - & - & 29.67 & 35.58 \\
SQNRopt & 17.55 & 23.57 & 29.59 & 35.61 \\
\hline
\end{tabular}

$D_{g}^{P U}\left(t_{i}^{*}\right)$, is very close to the granular distortion of the OC quantizer $\left(D_{g}^{\text {Ben }}\left(t_{i}^{*}\right)\right.$ or $\left.D_{g}^{\text {exact }}\left(t_{i}^{*}\right)\right)$. Therefore, the linearization of the inner regions has a very small influence on the increase of the granular distortion of the PU quantizer. But, from the last column of Table 1 we can see that the granular distortion in the interval $\left[-t_{16}^{*}, t_{16}^{*}\right]$ of the PU quantizer is significantly higher (for about $30 \%$ ) than the granular distortion of the OC quantizer. Therefore, we can conclude that the last region $\left[t_{15}^{*}, t_{16}^{*}\right]$ has the highest contribution on the increase of the granular distortion of the PU quantizer. Hence, the last region of the linearized PU quantizer should be optimized with the aim of the decrease of the granular distortion. In this paper, we propose two methods for optimization to improve the linearization of the last region. These methods for optimization are described in the next chapter.

\section{THE IMPROVEMENT OF THE LINEARIZATION BY OPITIMIZNG THE LAST REGION}

In this section, we will optimize the last region (the $L$ th region) $\left(t_{L-1}^{*}, t_{L}^{*}=x_{\max }^{*}\right]$, and give expressions for the maximum amplitude of the linearized quantizer $x_{\max }^{*}$ and the thresholdV of the granular region $t_{N-1}^{\prime}=t_{L, N / L-1}$. Optimizing these parameters, the distortion reduces and the quality of the output signal increases. In this paper, we propose two methods for optimization of $t_{N-1}^{\prime}$.

Method I. According to this method, the maximum amplitude of the linearized quantizer $x_{\max }^{*}$ is chosen to be equal to the maximum amplitude of the nonlinearized quantizer $x_{\max }^{*}$ given by (15). In this case, the size of the cell is equal to

$$
\Delta_{L}^{*}=\frac{x_{\max }^{*}-t_{L-1}^{*}}{N / L} .
$$

The threshold of the granular region is equal to

$$
t_{N-1}^{\prime}=x_{\max }^{*}-\Delta_{L}^{*}
$$

Method II. This method proposes that the threshold of the granular region $t_{N-1}^{\prime}$ is determined numerically, respecting the criterion of minimum total distortion DL for the last region (See Fig. 2), where the last representational level $y_{N}$ is centroid of the last cell $\left(t_{N-1}^{\prime}, \infty\right)[1]$

$$
y_{N}=\frac{\int_{t_{N-1}^{\prime}}^{\infty} x p(x) \mathrm{d} x}{\int_{t_{N-1}^{\prime}}^{\infty} p(x) \mathrm{d} x} .
$$

The value of the total distortion $D_{L}$, for the last region, in this case is

$$
D_{L}=\frac{\Delta_{L}^{*}}{12} \int_{t_{L-1}^{*}}^{t_{N-1}^{\prime}} p(x) \mathrm{d} x+\frac{1}{2} \exp \left(-\sqrt{2} t_{N-1}^{\prime}\right)
$$

where the size of the cell in the last L-th region is equal to

$$
\Delta_{L}^{*}=\frac{t_{N-1}^{\prime}-t_{L-1}^{*}}{N / L-1} .
$$

Changing (27) into (26), and numerically solving (26), $t_{N-1}^{\prime}$ is found.

\section{NUMERICAL RESULTS}

Numerical results presented in this section are obtained for the case when the total number of levels is equal to $N=128$, the number of regions is equal to $L=16$ and the number of cells inside the each region is equal to $N / L=8$. Table 2 shows the numerical results for the previously described methods of the optimization of the last region. Comparing the obtained values of $S Q N R$, one can notice that method II is actually improved method I. Optimizing the last region by using method II, value of $S Q N R$ is $35.34 \mathrm{~dB}$, that is very close to values of $S Q N R$ for nonlinear optimal compressor function $[7,8]$. The value of $S Q N R$ for the case where all representational levels are centroids and the optimization 
of the last region performed according to method II, is $35.35 \mathrm{~dB}$. Due to minor differences in the quality of the output signal and less complexity of coding and decoding process, this paper proposes method II as the best solution. The values of $S Q N R$ for different values of parameters $N$ and $L$, obtained on the basis of the method II are shown in Table 3 . The last row in Table 3 represents the optimal value of $S Q N R$ for different values of parameter $N[7,8]$. Taking into consideration simple and fast coding and decoding process and the less complexity of the system, and the quality of the output signal, one can conclude that the best result is achieved for values of parameters $L=16$ and $N=128$. By introducing the linearization, coding and decoding process is considerably simpler and faster. In order to linear model represents a worthy substitution for the original nonlinear model, the correct selection of an amplitude range of quantizer is important. Therefore it is necessary to optimize $S Q N R$ which is very difficult to achieve analytically because many parameters of the quantizer indirectly depend on the size of the amplitude range of the quantizer. Therefore, we decided for numerical determination of the amplitude range of quantizer respecting the criterion of minimum distortion (see Fig. 2).

\section{CONCLUSION}

This paper proposes the new method of the linearization of the optimal compressor function. This linearized quantizer can be considered as a generalized quantized, whose special cases are the nonuniform optimal companding quantizer (for $L=N$ ) and the uniform quantizer $(L=1)$. Our aim was to decrease the complexity of the nonuniform optimal companding quantizer by linearization, but to keep performances near to those of the optimal companding quantizer. Based on the results obtained by the adequate analysis of the proposed method for optimization of the last region, one can conclude that the proposed methods are very effective solution because obtained value of $S Q N R$ is close to that of the nonlinear optimal compressor function. Since method II represents the improvement of method I, in this paper, method II is proposed as the best solution. Also, it is shown that the highest signal quality, for the lowest complexity of the system, is achieved by the method II for the parameter values $L=16$ and $N=128$. For these values of parameters, the complexity is considerably reduced compared to the nonuniform optimal quantizer, but $S Q N R$ is only for $0.32 \mathrm{~dB}$ smaller. Also, the combination of parameters $L=32$ and $N=128$ can be used as a very good solution (this combination has a higher complexity than the previous combination but much smaller complexity than the nonuniform quantizer, but the $S Q N R$ is almost identical to $S Q N R$ of the nonuniform quantizer). This linearized model allows to us to choose the best combination of parameters, regarding the application and the memory and computation capacity.

\section{REFERENCES}

[1] JAYANT, N. S.-NOLL, P.: Digital Coding Of Waveforms, Principles and Applications to Speech and Video, Prentice Hall, New Jersey, 1984, Chapter 4, pp. 115-220.

[2] NIKOLIC, J.-PERIC, Z.-ANTIC, D.-JOVANOVIC, A.DENIC, D.: Low Complex Forward Adaptive Loss Compression Algorithm and its Aplication in Speech Coding, Journal of Electrical Engineering 62 No. 1 (2011), 19-24.

[3] PERIC, Z.-PETKOVIC, M.-DinCIC, M. : Simple Compression Algorithm for Memoryless Laplacian Source Based on the Optimal Companding Technique, Informatica 20 No. 1, 99-114.

[4] PERIC, Z.-NIKOLIC, J.: Analysis of Compressor Functions for Laplacian Source's Scalar Compandor Construction, Data Recording, Storage and Processing 8 No. 2, 15-24.

[5] MARCO, D.-NEUHOFF, D. L. : Low-Resolution Scalar Quantization for Gaussian and Laplacian Sources with Absolute and Squared Error Distortion Measures, Technical report, January, 2006.

[6] PERIC, Z.-NIKOLIC, J.-POKRAJAC, D.: Estimation of the Support Region for Laplacian Source Scalar Quantizer, Journal of Electrical Engineering 58 No. 1 (2007), 47-51.

[7] NA, S. : Asymptotic Formulas for Mismatched Fixed-Rate Minimum MSE Laplacian Quantizers, IEEE Signal Processing Letters 15, 13-16.

[8] NA, S. : On the Support of Fixed-Rate Minimum Mean-Squared Error Scalar Quantizers for a Laplacian Source, IEEE Trans. Information Theory $\mathbf{5 0}$ No. 5, 937-944.

Received 25 July 2011

Zoran H. Perić was born in Niš, Serbia, in 1964. He received the BS degree from the Faculty of Electronic Engineering, Niš, Serbia, in 1989, and MS degree from the University of Niš, in 1994. He received the PhD degree from the University of Niš, in 1999. He is currently a full Professor at the Department of Telecommunications and vice dean of the Faculty of Electronic Engineering, University of Niš, Serbia. His current research interests include the information theory, source and channel coding and signal processing. He is particularly working on scalar and vector quantization techniques in speech and image coding. He is author and coauthor of over 200 papers. He has been a reviewer for IEEE Transactions on Information Theory, IEEE Transactions on Signal Processing, IEEE Transactions on Communications, Informatica and The International Journal for Computation and Mathematics in Electrical Engineering (COMPEL), Electronics and Electrical Engineering.

Lazar Z. Velimirović was born in Prokuplje, Serbia, in 1983. He received the MS degree in electrical engineering from the Faculty of Electronic Engineering, University of Niš, Serbia, in 2008, and $\mathrm{PhD}$ degree from the University of Niš, in 2013. He is currently employed as a research associate at the Mathematical Institute of the Serbian Academy of Sciences and Arts, Belgrade, Serbia.

Milan R. Dinčić was born in Niš, Serbia, in 1983. He received the BS degree from the Faculty of Electronic Engineering, Niš, Serbia, in 2007, and PhD degree from the University of Niš, in 2012. He is now with the Faculty of Electronic Engineering at the University of Niš. His current research interests include sensors and measurements, information theory, source and channel coding, signal processing, scalar and vector quantization. He is author and coauthor of about 25 scientific papers. 